\title{
On Edge-Disjoint Empty Triangles of Point Sets *
}

\author{
J. Cano Vila ${ }^{\dagger} \quad$ L.F. Barba ${ }^{\ddagger} \quad$ J. Urrutia ${ }^{\S} \quad$ T. Sakai
}

October 25, 2011

\begin{abstract}
Let $P$ be a set of points in the plane in general position. Any three points $x, y, z \in P$ determine a triangle $\Delta(x, y, z)$ of the plane. We say that $\Delta(x, y, z)$ is empty if its interior contains no element of $P$. In this paper we study the following problems: What is the size of the largest family of edge-disjoint triangles of a point set? How many triangulations of $P$ are needed to cover all the empty triangles of $P$ ? We also study the following problem: What is the largest number of edge-disjoint triangles of $P$ containing a point $q$ of the plane in their interior? We establish upper and lower bounds for these problems.
\end{abstract}

\section{Introduction}

Let $P$ be a set of $n$ points in the plane in general position. A geometric graph on $P$ is a graph $G$ whose vertices are the elements of $P$, two of which are adjacent if they are joined by a straight line segment. We say that $G$ is plane if it has no edges that cross each other. A triangle of $G$ consists of three points $x, y, z \in P$ such that $x y, y z$, and $z x$ are edges of $G$; we will denote it as $\Delta(x, y, z)$. If in addition $\Delta(x, y, z)$ contains no elements of $P$ in its interior, we say that it is empty.

*Partially supported by projects MTM2009-07242, and by projects MTM2006-03909 (Spain), and SEP-CONACYT of Mexico, Proyecto 80268.

${ }^{\dagger}$ Posgrado en Ciencia e Ingeniería de la Computación, Universidad Nacional Autónoma de México, j_cano@uxmcc2.iimas.unam.mx

${ }^{\ddagger}$ Posgrado en Ciencia e Ingeniería de la Computación, Universidad Nacional Autónoma de México, 1.barba@uxmcc2.iimas.unam.mx

§Instituto de Matemáticas, Universidad Nacional Autónoma de México, urrutia@ matem. unam.mx

๑ Research Institute of Educational Development, Tokai University, sakai@tokai-u.jp 
In a similar way, we say that if $x, y, z \in P$, then $\Delta(x, y, z)$ is a triangle of $P$, and that $x y, y z$, and $z x$ are the edges of $\Delta(x, y, z)$. If $\Delta(x, y, z)$ is empty, it is called a 3 -hole of $P$. A 3-hole of $P$ can be thought of as an empty triangle of the complete geometric graph $\mathcal{K}_{\mathcal{P}}$ on $P$. We remark that $\Delta(x, y, z)$ will denote a triangle of a geometric graph, and also a triangle of a point set.

A well-known result in graph theory says that for $n=6 k+1$, or $n=$ $6 k+3$, the edges of the complete graph $K_{n}$ on $n$ vertices can be decomposed into a set of $\left(\begin{array}{l}n \\ 2\end{array}\right) / 3$ edge-disjoint triangles. These decompositions are known as Steiner triple systems [23]; see also Kirkman's schoolgirl problem [17, 22]. In this paper, we address some variants of that problem, but for geometric graphs.

Given a point set $P$, let $\delta(P)$ be the size of the largest set of edge-disjoint empty triangles of $P$. It is easy to see that for point sets in convex position with $n=6 k+1$ or $n=6 k+3$ elements, $\delta(P)=\left(\begin{array}{l}n \\ 2\end{array}\right) / 3$. Indeed any triangle of $P$ is empty, and the problem is the same as that of decomposing the edges of the complete geometric graph $\mathcal{K}(P)$ on $P$ into edge-disjoint triangles. On the other hand, we prove that for some point sets, namely Horton point sets, $\delta(P)$ is $O(n \log n)$.

We then study the problem of covering the empty triangles of point sets with as few triangulations of $P$ as possible. For point sets in convex position, we prove that we need essentially $\left(\begin{array}{l}n \\ 3\end{array}\right) / 4$ triangulations; our bound is tight. We also show that there are point sets $P$ for which $O(n \log n)$ triangulations are sufficient to cover all the empty triangles of $P$ for a given point set $P$.

Finally, we consider the problem of finding a point $q$ not in $P$ contained in the interior of many edge-disjoint triangles of $P$. We prove that for any point set, there is a point $q \notin P$ contained in at least $n^{2} / 12$ edge-disjoint triangles. Furthermore, any point in the plane, not in $P$, is contained in at most $n^{2} / 9$ edge-disjoint triangles of $P$, and this bound is sharp. In particular, we show that this bound is attained when $P$ is the set of vertices of a regular polygon.

\subsection{Preliminary work}

The study of counting and finding $k$-holes in point sets has been an active area of research since Erdős and Szekeres [11, 12] asked about the existence of $k$-holes in planar point sets. It is known that any point set with at least ten points contains 5-holes; e.g. see [14]. Horton [15] proved that for $k \geq 7$ there are point sets containing no $k$-holes. The question of the existence of 6-holes remained open for many years, but recently Nicolás [19] proved that any point set with sufficiently many points contains a 6-hole. A second 
proof of this result was subsequently given by Gerken [13].

The study of properties of the set of triangles generated by point sets on the plane has been of interest for many years. Let $f_{k}(n)$ be the minimum number of $k$-holes that a point set has. Clearly a point set has a minimum of $f_{3}(n)$ empty triangles. Katchalski and Meir [16] proved that $\left(\begin{array}{l}n \\ 2\end{array}\right) \leq f_{3}(n) \leq$ $c n^{2}$ for some $c<200$; see also Purdy [21]. Their lower bounds were improved by Dehnhardt [9] to $n^{2}-5 n+10 \leq f_{3}(n)$. He also proved that $\left(\begin{array}{c}n-3 \\ 2\end{array}\right)+6 \leq$ $f_{4}(n)$. Point sets with few $k$-holes for $3 \leq k \leq 6$ were obtained by Bárány and Valtr [2]. The interested reader can read [18] for a more accurate picture of the developments in this area of research.

Chromatic variants of the Erdös-Szekeres problem have recently been studied by Devillers, Hurtado, Károly, and Seara [10]. They proved among other results that any bi-chromatic point set contains at least $\frac{n}{4}-2$ compatible monochromatic empty triangles. Aichholzer et al. [1] proved that any bi-chromatic point set always contains $\Omega\left(n^{5 / 4}\right)$ empty monochromatic triangles; this bound was improved by Pach and Tóth [20] to $\Omega\left(n^{4 / 3}\right)$.

\section{Sets of edge-disjoint empty triangles in point sets}

Let $P$ be a set of points in the plane, and $\delta(P)$ the size of the largest set of edge-disjoint empty triangles of the complete graph $\mathcal{K}(P)$ on $P$. In this section we study the following problem:

Problem 1. How small can $\delta(P)$ be?

We show that if $P$ is a Horton set, then $\delta(P)$ is $O(n \log n)$. On the other hand, it follows directly from Theorem 7 that if $P$ is the set of vertices of a regular polygon then $\delta(P)$ is at least $\frac{n^{2}}{9}-n$.

For any integer $k \geq 1$, Horton [15] recursively constructed a family of point sets $H_{k}$ of size $2^{k}$ as follows:

(a) $H_{1}=\{(0,0),(1,0)\}$.

(b) $H_{k}$ consists of two subsets of points $H_{k-1}^{-}$and $H_{k-1}^{+}$obtained from $H_{k-1}$ as follows: If $p=(i, j) \in H_{k-1}$, then $p^{\prime}=(2 i, j) \in H_{k-1}^{-}$and $p^{\prime \prime}=\left(2 i+1, j+d_{k}\right) \in H_{k-1}^{+}$. The value $d_{k}$ is chosen large enough such that any line $\ell$ passing through two points of $H_{k-1}^{+}$leaves all the points of $H_{k-1}^{-}$below it; see Figure 1 . 

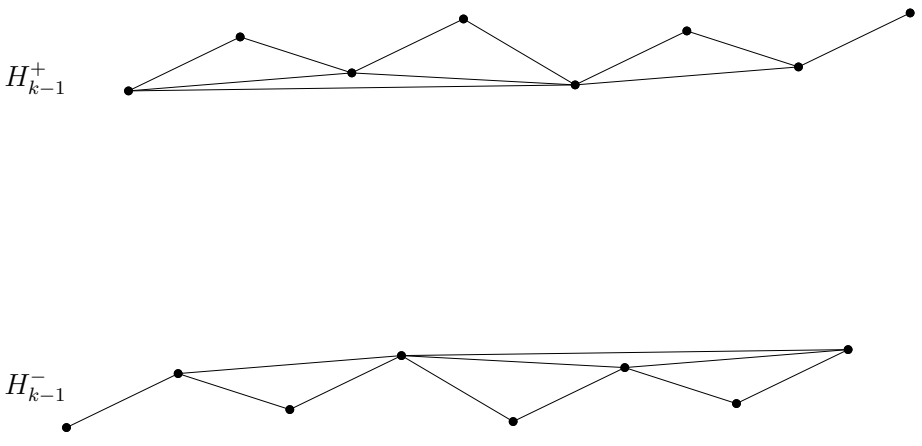

Figure 1: $H_{4}$. The edges of $H_{3}^{+}$(resp. $H_{3}^{-}$) visible from below (resp. above), are shown.

We say that a line segment $p q$ joining two elements $p$ and $q$ of $H_{k}$ is visible from below (resp. above) if there is no point of $H_{k}$ below it (resp. above it); that is there is no element $r$ of $H_{k}$ such that the vertical line through $r$ intersects $p q$ above $r$ (resp. below $r$ ). Let $B\left(H_{k}\right)$ be the set of line segments of $H_{k}$ visible from below. The following result which we will use later was proved by Bárány and Valtr in [2]; see also [3]:

Lemma 1. $\left|B\left(H_{k}\right)\right|=2^{k+1}-(k+2)$.

The following result is proved in [3] by using this lemma:

Theorem 1. For every $n=2^{k}, k \geq 1$, there is a point set (namely $H_{k}$ ) such that there is a geometric graph on $H_{k}$ with $\left(\begin{array}{l}n \\ 2\end{array}\right)-O(n \log n)$ edges with no empty triangles.

In other words, it is always possible to remove $O(n \log n)$ edges from the complete graph $\mathcal{K}_{H_{k}}$ in such a way that the remaining graph contains no empty triangles. The main idea is that by removing from $\mathcal{K}_{H_{k}}$ all the edges of $H_{k-1}^{+}$(respectively $H_{k-1}^{-}$) visible from below (respectively above), no empty triangle remains with vertices in both $H_{k-1}^{+}$, and $H_{k-1}^{-}$.

Observe now that if a geometric graph has $k$ edge-disjoint empty triangles, then we need to take at least $k$ edges away from $G$ for the graph that remains to contain no empty triangles. It follows now that the complete graph $\mathcal{K}_{H_{k}}$ has at most $O(n \log n)$ edge-disjoint empty triangles. Thus we have proved:

Theorem 2. There is a point set, namely $H_{k}$, such that any set of edgedisjoint empty triangles of $H_{k}$ contains at most $O(n \log n)$ elements. 
Clearly for any point set $P$, the size of the largest set of edge-disjoint triangles of $P$ is at least linear. We conjecture:

Conjecture 1. Any point set $P$ in general position always contains a set with at least $O(n \log n)$ edge-disjoint empty triangles.

\section{Covering the triangles of point sets with trian- gulations}

An empty triangle $t$ of a point set $P$ is covered by a triangulation $T$ of $P$ if one of the faces of $T$ is $t$. In this section we consider the following problem:

Problem 2. How many triangulations of a point set are needed such that each empty triangle of $P$ is covered by at least one triangulation?

This problem, which is interesting on its own right, will help us in finding point sets for which $\delta(P)$ is large. We start by studying Problem 2 for point sets in convex position, and then for point sets in general position.

\subsection{Points in convex position}

All point sets $P$ considered in this subsection will be assumed to be in convex position, and their elements labeled $\left\{p_{0}, \ldots, p_{n-1}\right\}$ in counter-clockwise order around the boundary of $\mathrm{CH}(P)$. Since any triangulation of a point set of $n$ points in convex position corresponds to a triangulation of a regular polygon with $n$ vertices, solving Problem 2 for point sets in convex position is equivalent to solving it for point sets whose elements are the vertices of a regular polygon. Suppose then that $P$ is the set of vertices of a regular polygon, and that $c$ is the center of such a polygon.

A triangle is called an acute triangle if all of its angles are smaller than $\frac{\pi}{2}$. We recall the following result in elementary geometry given without proof.

Observation 1. A triangle with vertices in $P$ is acute if and only if it contains $c$ in its interior.

The following result is relatively well known:

Lemma 2. Let $P$ be the set of vertices of a regular $n$-gon $Q$, and $c$ the center of $Q$. Then:

- If $n$ is even, $c$ is contained in the interior of $\frac{1}{4}\left[\left(\begin{array}{l}n \\ 3\end{array}\right)-\frac{n(n-2)}{2}\right]$ acute triangles of $P$. 

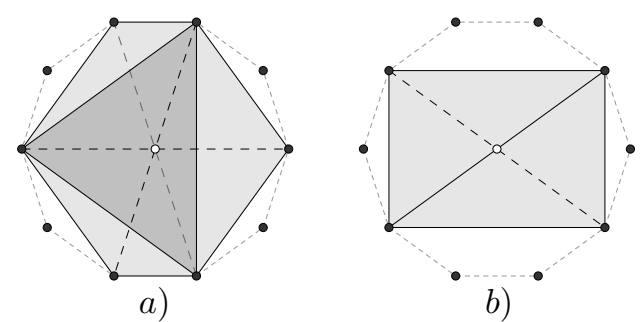

Figure 2: $a$ ) Constructing $t_{4}(i, j, k)$, and $\left.b\right)$ pairing triangles sharing an edge which contains $c$ in the middle.

- If $n$ is odd, $c$ is contained in $\left[\left(\begin{array}{l}n \\ 3\end{array}\right)-\frac{n(n-1)(n-3)}{8}\right]=\frac{1}{4}\left[\left(\begin{array}{l}n \\ 3\end{array}\right)+\frac{n(n-1)}{2}\right]$ acute triangles of $P$.

Let $f(n)=\frac{1}{4}\left[\left(\begin{array}{l}n \\ 3\end{array}\right)+\frac{n(n-2)}{2}\right]$ for $n$ even, and $f(n)=\frac{1}{4}\left[\left(\begin{array}{l}n \\ 3\end{array}\right)+\frac{n(n-1)}{2}\right]$ for $n$ odd. We now prove:

Theorem 3. $f(n)$ triangulations are always sufficient, and always necessary, to cover all the triangles of a regular polygon.

Proof. Suppose first that $n$ is even. For each vertex $p_{i}$ of $P$, let $\alpha\left(p_{i}\right)=p_{i+\frac{n}{2}}$ be the antipodal vertex of $p_{i}$ in $P$, where addition is taken mod $n$. Suppose that $\Delta\left(p_{i}, p_{j}, p_{k}\right)$ is an acute triangle of $P$ (i.e. it contains $c$ in its interior), $i<j<k$. Let $t_{4}(i, j, k)$ be the following set of four triangles:

$t_{4}(i, j, k)=\left\{\Delta\left(p_{i}, p_{j}, p_{k}\right), \Delta\left(\alpha\left(p_{i}\right), p_{j}, p_{k}\right), \Delta\left(p_{i}, \alpha\left(p_{j}\right), p_{k}\right), \Delta\left(p_{i}, p_{j}, \alpha\left(p_{k}\right)\right)\right\} ;$

see Figure 2 a).

It is easy to see that all the triangles of $P$ except those that have a right angle are in

$$
\bigcup t_{4}(i, j, k)
$$

where $i, j, k$ range over all triples such that $\Delta\left(p_{i}, p_{j}, p_{k}\right)$ contains $c$ in its interior.

On the other hand, it is easy to see that if a triangle $t$ of $P$ contains $c$ in the middle of one of its edges (clearly $t$ is a right triangle), this edge joins two antipodal vertices of $P$; see Figure $2 \mathrm{~b}$ ). Thus we have exactly

$$
\frac{n}{2} \times(n-2)
$$


such triangles. It is easy to find

$$
\frac{n(n-2)}{4}
$$

triangulations of $P$ such that each of them cover two of these triangles. Since each triangulation of $P$ contains exactly one acute triangle of $P$ or two triangles sharing an edge that contains $c$ at its middle point, it follows that

$$
\frac{1}{4}\left[\left(\begin{array}{l}
n \\
3
\end{array}\right)-\frac{n(n-2)}{2}\right]+\frac{n(n-2)}{4}=\frac{1}{4}\left[\left(\begin{array}{l}
n \\
3
\end{array}\right)+\frac{n(n-2)}{2}\right]
$$

triangulations are necessary and sufficient to cover all the triangles of $P$. To show that this number of triangulations are needed, we point out that any two acute triangles of $P$ cannot belong to the same triangulation (note that they intersect at $c$ ). Moreover these triangulations are different from those containing right triangles. Our result follows.

A similar argument follows for $n$ odd, except that some extra care has to be paid to the way in which we group the non-acute triangles of $P$ around the acute triangles of $P$.

Thus the number of triangulations needed to cover all the triangles of $P$ is asymptotically $\left(\begin{array}{l}n \\ 3\end{array}\right) / 4$. The next result follows trivially:

Corollary 1. Let $P$ be a set of $n$ points in convex position, and $p$ any point in the interior of $C H(P)$. Then $p$ belongs to the interior of at most $\frac{\left(\begin{array}{l}n \\ 3\end{array}\right)}{4}+O\left(n^{2}\right)$ triangles of $P$.

\subsection{Covering the empty triangles on the Horton set}

We will now show that all the empty triangles in $H_{k}$ can be covered with $O(n \log n)$ triangulations. The bound is tight.

Consider an edge $e$ of $H_{k}$ that is visible from below, and a vertical line $\ell$ that intersects $e$ at a point $q$ in the interior of $e$. The depth of $e$ is the number of edges of $H_{k}$, visible from below, intersected by $\ell$ below $q$. It is not hard to see that the maximal depth of an edge of $H_{k}$ visible from below is at most $\log n-1$, and that this bound is tight; see Figure 3. Moreover, it is easy to see that the union of all edges of $H_{k}$ with the same depth is an $x$-monotone path. Now we can prove:

Theorem 4. $\Theta(n \log n)$ triangulations of $H_{k}$ are necessary and sufficient to cover the set of empty triangles of $H_{k}$. 


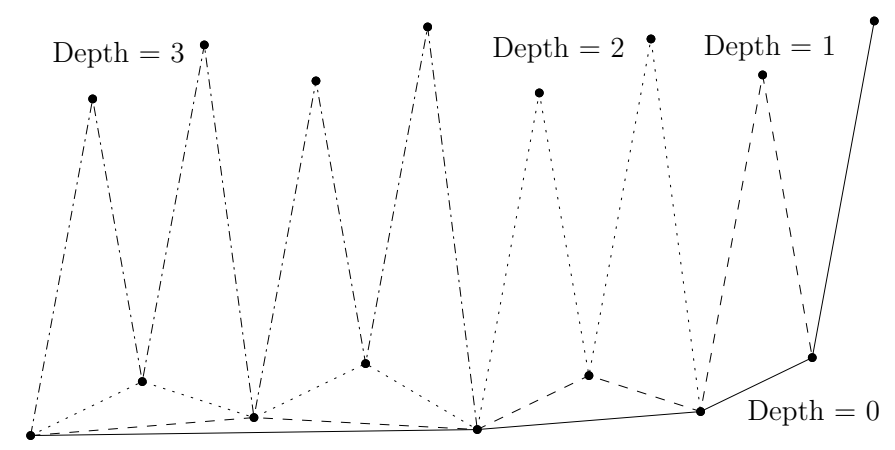

Figure 3: The depth of an edge.

Proof. Consider the sets $H_{k-1}^{+}$and $H_{k-1}^{-}$. We will show how to cover all the empty triangles of $H_{k}$ with two vertices in $H_{k-1}^{+}$and one in $H_{k-1}^{-}$with $O(n \log n)$ triangulations. Label the elements of $H_{k-1}^{-}$from left to right as $p_{0}, \ldots, p_{\frac{n}{2}-1}$.

For each $0 \leq d \leq k-1$, proceed as follows: For every $p_{j} \in H_{k-1}^{-}$join $p_{j}$ to the endpoints of all the edges of $H_{k-1}^{+}$of depth $d$. This gives us a set $I D_{d, j}^{+}$of interior-disjoint empty triangles. It is not hard to see that if $(d, j) \neq\left(d^{\prime}, j^{\prime}\right)$, then $I D_{d, j}^{+} \cap I D_{d^{\prime}, j^{\prime}}^{+}=\emptyset$.

It is easy to see that the union of these sets covers all the empty triangles with two vertices in $H_{k-1}^{+}$and one in $H_{k-1}^{-}$. In a similar way, cover all the triangles with two vertices in $H_{k-1}^{-}$, and one in $H_{k-1}^{+}$with a family of sets $I D_{d, j}^{-}$.

Let $\ell_{1}$ be the straight line connecting the leftmost point in $H_{k-1}^{+}$to the rightmost point in $H_{k-1}^{-}$, and $\ell_{2}$ the straight line that connects the rightmost point in $H_{k-1}^{+}$with the leftmost point of $H_{k-1}^{-}$. Let $q$ be a point slightly above the intersection point of $\ell_{1}$ with $\ell_{2}$.

It is clear that for each $I D_{d, j}^{+}$there is exactly one empty triangle that contains $q$ in its interior. This implies that $q$ is contained in $\Omega(n \log n)$ empty triangles and thus $\Omega(n \log n)$ triangulations are necessary to cover all the empty triangles in $H_{k}$.

Now we show that $O(n \log n)$ of $H_{k}$ triangulations are sufficient. Consider each set $I D_{d, j}^{+}$and $I D_{d, j}^{-}$, and complete it to a triangulation. This gives us $O(n \log n)$ triangulations that cover all the triangles with vertices in both $H_{k-1}^{+}$and $H_{k-1}^{-}$.

Take a set of triangulations $\mathcal{T}_{k-1}^{+}=\left\{T_{1}^{+}, \ldots, T_{m}^{+}\right\}$of $H_{k-1}^{+}$that covers all 
of its empty triangles. Since $H_{k-1}^{+}$and $H_{k-1}^{-}$are isomorphic, we can find a set of triangulations $\mathcal{T}_{k-1}^{-}=\left\{T_{1}^{-}, \ldots, T_{m}^{-}\right\}$of $H_{k-1}^{-}$that covers all the empty triangles of $H_{k-1}^{-}$such that $T_{i}^{+}$is isomorphic to $T_{i}^{-}$. For each $i$, we can find a triangulation $T_{i}$ of $H_{k}$ that contains $T_{i}^{+}$and $T_{i}^{-}$as induced subgraphs.

Thus if $T(n)$ is the number of triangulations required to cover the empty triangles of $H_{k}$, the following recurrence holds for $n=2^{k}$ :

$$
T(n)=T\left(\frac{n}{2}\right)+O(n \log n) .
$$

This solves to $T(n)=O(n \log n)$, and our result follows.

We conclude this section with the following conjecture:

Conjecture 2. At least $\Omega(n \log n)$ triangulations are needed to cover all the empty triangles of any point set with $n$ points.

\section{A point in many edge-disjoint triangles}

The problem of finding a point contained in many triangles of a point set was solved by Boros and Füredi [4], see also Bukh [6]. They proved:

Theorem 5. For any set $P$ of $n$ points in general position, there is a point in the interior of the convex hull of $P$ contained in $\frac{2}{9}\left(\begin{array}{l}n \\ 3\end{array}\right)+O\left(n^{2}\right)$ triangles of $P$. The bound is tight.

We now study a variant to this problem, in which we are interested in finding a point in many edge-disjoint triangles. We consider the following:

Problem 3. Let $P$ be a set of points in the plane in general position, and $q \notin P$ a point of the plane. What is the largest number of edge-disjoint triangles of $P$ such that $q$ belongs to the interior of all of them?

We start by giving some preliminary results, and then we study Problem 3 for point sets in general position, and sets of vertices of regular polygons.

Given a point set $P$, and a point $q$ not in $P$, let $\mathcal{T}(P, q)$ (or $\mathcal{T}(q)$ for short) be the set of triangles of $P$ that contain $q$. We define the graph $G(P, q)$ whose vertex set is $\mathcal{T}(q)$ in which two triangles are adjacent if they share an edge; see Figure 4 . We may assume that $q$ does not belong to any line passing through two elements of $P$. We now prove:

Lemma 3. The degree of every vertex of $G(P, q)$ is exactly $n-3$. 



Figure 4: $G(P, q)$.

Proof. Let $\Delta(x, y, z)$ be a triangle that contains $q$ in its interior. Let $p$ be any point in $P \backslash\{x, y, z\}$. Then exactly one of the triangles $\Delta(x, y, p)$, $\Delta(x, p, z)$, or $\Delta(p, y, z)$ contains $q$; see Figure 5 . That is, exactly one of $\Delta(x, y, p), \Delta(x, p, z)$, or $\Delta(p, y, z)$ belongs to $\mathcal{T}(q)$. Our result follows.

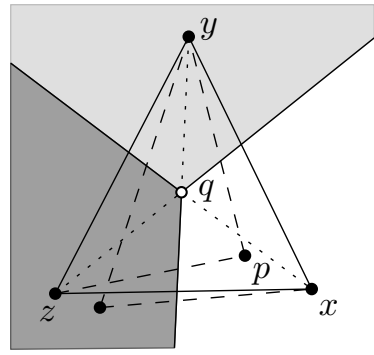

Figure 5:

Observe now that finding sets of edge-disjoint triangles that contain $q$ is equivalent to finding independent sets in $G(P, q)$. Let $\tau(P, q)$ (or $\tau(q)$ for short) be the largest number of edge-disjoint triangles on $P$ containing $q$. We now prove:

\section{Lemma 4.}

$$
\frac{|\mathcal{T}(q)|}{n-2} \leq \tau(q) \leq \frac{3|\mathcal{T}(q)|}{n} .
$$

Proof. It follows from Lemma 3 that the size of the largest independent set of $G(P, q)$ is at least $\frac{|\mathcal{T}(q)|}{n-2}$. To prove our upper bound, it is sufficient to 
observe that if a vertex of $G(P, q)$ is not in an independent set $I$ of $G(P, q)$, then it is adjacent to at most three vertices in it, one per each of its edges. Hence by counting the number of edges connecting a vertex in $I$ to another in $\mathcal{T}(q) \backslash I$, we obtain that:

$$
(n-3)|I| \leq 3|\mathcal{T}(q) \backslash I|
$$

Our result follows.

From Theorem 5 and Lemma 4 it is easy to see that in any set of $n$ points in general position on the plane there is a point $q$ such that

$$
\frac{n^{2}}{27}+O(n) \approx \frac{\frac{2}{9}\left(\begin{array}{l}
n \\
3
\end{array}\right)+O\left(n^{2}\right)}{n-2} \leq \tau(q) \leq \frac{3 \cdot \frac{2}{9}\left(\begin{array}{l}
n \\
3
\end{array}\right)+O\left(n^{2}\right)}{n} \approx \frac{n^{2}}{9}+O(n) .
$$

Thus we have:

Corollary 2. For any point set in general position on the plane there is a point $q$ such that $\tau(q) \leq \frac{n^{2}}{9}+O(n)$.

We now prove an even stronger result. We now prove:

Proposition 1. Let $P$ a set of $n$ points in general position on the plane. Then for any point $q \notin P$ of the plane $\tau(q) \leq n^{2} / 9$.

Proof. Let $q \notin P$ be any point of the plane. If $q$ is on a straight line passing through two elements of $P$, then by slightly moving it, $q$ could be moved to a position in which it is contained in more edge-disjoint triangles. Thus assume that $q$ is not on any straight line through two elements of $P$.

First we show the following lemma:

Lemma 5. There exist three straight lines passing through $q$ such that they partition $P$ into six subsets $P_{0}, P_{1}, \ldots, P_{5}$ in counter-clockwise order around $q$, with $\left|P_{0}\right|=\left|P_{2}\right|=\left|P_{4}\right|$ (we allow the possibility that $P_{i}=\emptyset$ for some $i$ ).

Proof. Let $l_{0}$ be a straight line passing through $q$ such that one of the halfplanes bounded by $l_{0}$, say the one on top of it, contains an even number of elements of $P$. Take other straight lines $l_{1}$ and $l_{2}$ passing through $q$, and define the subsets $P_{i}$ of $P, 0 \leq i \leq 5$, as shown in Figure 6 a), where $\left|P_{0} \cup P_{1} \cup P_{2}\right|$ is even. Let $l^{*}$ be a straight line passing through $q$, equipartitioning the elements of $P_{0} \cup P_{1} \cup P_{2}$.

Choose $l_{1}$ and $l_{2}$ such that initially $\left|P_{0}\right|=\left|P_{2}\right|=\left|P_{3}\right|=\left|P_{5}\right|=0$. From their initial positions, rotate $l_{1}$ counter-clockwise and $l_{2}$ clockwise around $q$ 




a)

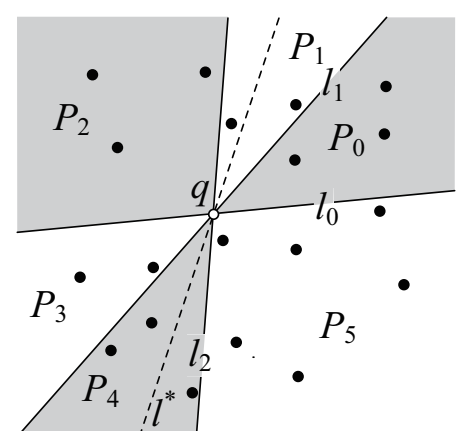

b)

Figure 6: Partitions of $P$.

in such a way that $P_{0}$ and $P_{2}$ always contain the same number of elements, and until they both reach the position of $l^{*}$ at the same time, and the boundary of $P_{4}$ always contains no more than one element of $P$.

Initially $\left|P_{4}\right| \geq 0=\left|P_{0}\right|$. On the other hand, we have $\left|P_{4}\right|=0 \leq\left|P_{0}\right|$ when $l_{1}$ and $l_{2}$ reach the position of $l^{*}$. Hence at some point while rotating $l_{1}$ and $l_{2}$, we have that $\left|P_{0}\right|=\left|P_{2}\right|=\left|P_{4}\right|$; see Figure $6 \mathrm{~b}$ ).

Let $P_{0}, P_{1}, \ldots, P_{5}$ be as in Lemma 5 . Write $\left|P_{i}\right|=n_{i}$ for $0 \leq i \leq 5$ (we have $n_{0}=n_{2}=n_{4}$ ). We henceforth read indices modulo 6 . Let $\mathcal{T}$ be a set of edge-disjoint triangles with vertices in $P$, containing $q$ in its interior. For integers $i, j, k$, let $\mathcal{T}_{i j k}$ denote the set of elements of $\mathcal{T}$ such that it has one vertex in $P_{i}$, another in $P_{j}$ and the other in $P_{k}$, and let $t_{i j k}=\left|\mathcal{T}_{i j k}\right|$; see Figure 7.



a)

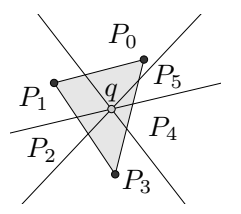

b)

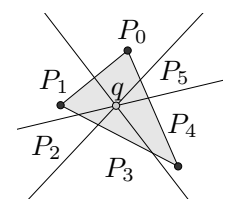

c)

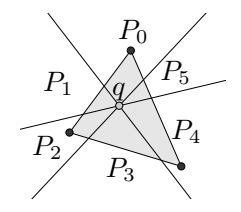

d)

Figure 7: Triangles in the $\mathcal{T}_{i j k}$ 's. 


$$
\begin{aligned}
\mathcal{T} & =\left[\cup_{i=0}^{5} \mathcal{T}_{i i(i+3)}\right] \cup\left[\cup_{i=0}^{5} \mathcal{T}_{i(i+1)(i+3)}\right] \cup\left[\cup_{i=0}^{5} \mathcal{T}_{i(i+1)(i+4)}\right] \cup\left[\cup_{i=0}^{5} \mathcal{T}_{i(i+2)(i+4)}\right] \\
& =\left[\cup_{i=0}^{5} \mathcal{T}_{i i(i+3)}\right] \cup\left[\cup_{i=0}^{5} \mathcal{T}_{i(i+2)(i+5)}\right] \cup\left[\cup_{i=0}^{5} \mathcal{T}_{i(i+2)(i+3)}\right] \cup\left[\cup_{i=0}^{5} \mathcal{T}_{i(i+2)(i+4)}\right] .
\end{aligned}
$$

$$
\begin{aligned}
\sum_{i=0}^{5} f(i)+2 \sum_{i=0}^{2} g(i)= & \sum_{i=0}^{5}\left(t_{i(i+2)(i+3)}+t_{i(i+2)(i+4)}+t_{i(i+2)(i+5)}\right) \\
& +2 \sum_{i=0}^{5}\left(t_{i(i+2)(i+3)}+t_{i(i+2)(i+5)}\right)+4 \sum_{i=0}^{5} t_{i i(i+3)} \\
= & 3|\mathcal{T}|+\sum_{i=0}^{5} t_{i i(i+3)} \geq 3|\mathcal{T}|
\end{aligned}
$$

$$
\sum_{i=0}^{5} f(i)+2 \sum_{i=0}^{2} g(i) \leq \sum_{i=0}^{5} n_{i} n_{i+2}+2 \sum_{i=0}^{2} n_{i} n_{i+3} .
$$

On the other hand, if we denote the right-hand side of (3) by $S$,

$$
\begin{aligned}
& S=\left(n_{0} n_{2}+n_{2} n_{4}+n_{4} n_{0}\right)+\left(n_{1} n_{3}+n_{3} n_{5}+n_{5} n_{1}\right) \\
& +2\left(n_{0} n_{3}+n_{2} n_{5}+n_{4} n_{1}\right) \\
& =\frac{l^{2}}{3}+\frac{2 l m}{3}+\left(n_{1} n_{3}+n_{3} n_{5}+n_{5} n_{1}\right) \text {, }
\end{aligned}
$$


a) $n_{5}$ for some partition (Figure 8).

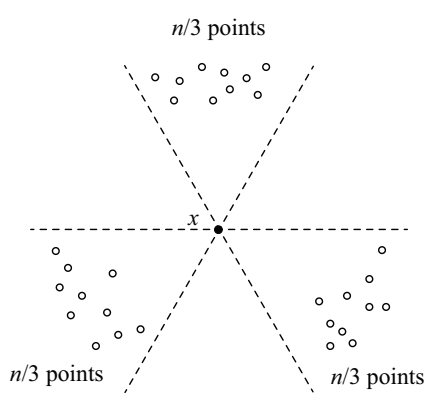

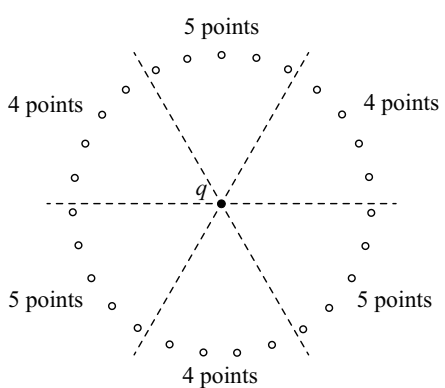

b)

Figure 8: A vertex set of a regular 27-gon.

316

317

$327 \quad n / 6-1$ elements of $P$.

We now prove: $\tau(q) \geq \frac{n^{2}}{12}$. given $P$ :

3 Now combining (3), (4) and (6), we obtain $|\mathcal{T}| \leq n^{2} / 9$, as desired.

To achieve the equality, it is necessary that $n_{0}=n_{2}=n_{4}$ and $n_{1}=n_{3}=$

Proposition 2. Let $n$ be a positive integer and $P$ a set of $n$ points in general position on the plane. Then there exists a point $q$ on the plane such that

Proof. We use the following lemma which was proved by Ceder [7] (see also [5]), and applied by Bukh [6] to obtain a lower bound of $\max _{q}|\mathcal{T}(q)|$ for

Lemma 6. There exist three straight lines such that they intersect at a point $q$ and partition the plane into 6 open regions each of which contains at least 
Let $q$ be as in Lemma 6 . We may assume that $q$ is not on any straight line passing through two elements of $P$. Let $m=\lceil n / 6\rceil-1$ and denote by $D_{0}, D_{1}, \ldots, D_{5}$ the six regions in counter-clockwise order around $q$. For each $0 \leq i \leq 5$, let $P_{i}$ be a subset of $P \cap D_{i}$ with $\left|P_{i}\right|=m$; see Figure 9 .

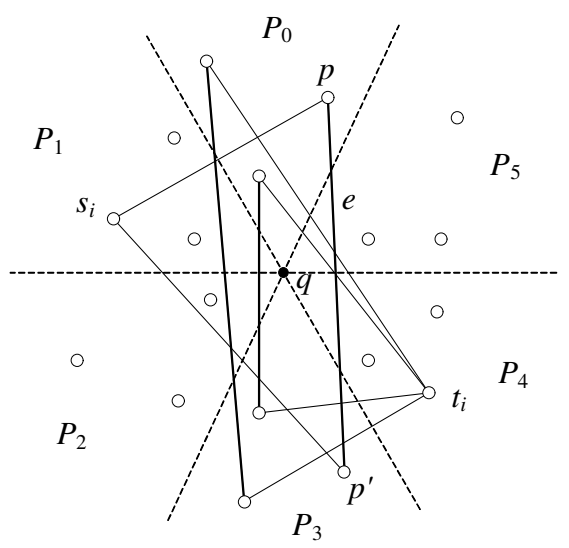

Figure 9: Matching $M_{i}$ (bold lines) and triangles using edges of $M_{i}$.

Now consider the geometric complete bipartite graph with vertex set $P_{0} \cup P_{3}$ and edge set $E=\left\{p p^{\prime} \mid p \in P_{0}, p^{\prime} \in P_{3}\right\}$. As a consequence of a well-known result in graph theory, $E$ can be decomposed into $m$ subsets $M_{i}$, $0 \leq i \leq m-1$, such that each $M_{i}$ is a perfect matching, i.e., consisting of $m$ independent edges. Let $P_{1}=\left\{s_{1}, s_{2}, \ldots, s_{m}\right\}$ and $P_{4}=\left\{t_{1}, t_{2}, \ldots, t_{m}\right\}$. For each $i$ and each element $e=p p^{\prime} \in M_{i}$, where $p \in P_{0}$ and $p^{\prime} \in P_{3}$, let $u_{i}$ denote either $s_{i}$ or $t_{i}$ according to whether $p p^{\prime} \cap D_{1}=\emptyset$ or $p p^{\prime} \cap D_{4}=\emptyset$. Then $\triangle\left(p, p^{\prime}, u_{i}\right)$ contains $q$ in its interior. Observe that all of the $m$ triangles in $\mathcal{T}_{i}=\left\{\triangle\left(p, p^{\prime}, u_{i}\right) \mid e=p p^{\prime} \in M_{i}\right\}$ are edge-disjoint, and all of the $m^{2}$ triangles in $\mathcal{T}_{03}=\cup_{i=0}^{m} \mathcal{T}_{i}$ are edge-disjoint as well.

Define the sets $\mathcal{T}_{14}$ and $\mathcal{T}_{25}$ of triangles similarly (the elements of $\mathcal{T}_{14}$ are triangles with one vertex in $P_{1}$, another in $P_{4}$ and the other in $P_{2} \cup P_{5}$, while the elements of $\mathcal{T}_{25}$ are triangles with one vertex in $P_{2}$, another in $P_{5}$ and the other in $\left.P_{3} \cup P_{0}\right)$. It can be observed that all of the $3 m^{2}=n^{2} / 12-O(n)$ triangles in $\mathcal{T}_{03} \cup \mathcal{T}_{14} \cup \mathcal{T}_{25}$ are edge-disjoint.

Thus by using Corollary 2, Proposition 1, and Proposition 2 we have:

Theorem 6. In any point set in general position, there is a point $q$ such that $\frac{n^{2}}{12} \leq \tau(q) \leq \frac{n^{2}}{9}$. Moreover, for any $q, \tau(q) \leq \frac{n^{2}}{9}$. 


\subsection{Regular Polygons}

By Theorem 6, any point in the interior of the convex hull of a point set is contained in at most $n^{2} / 9$ edge-disjoint triangles of $P$. It is also easy to construct point sets for which that bound is tight; see Figure 8 a). In fact, the point sets in that figure can be chosen in convex position.

We now show that the bound in Theorem 6 is also achieved when $P$ is the set of vertices of a regular polygon. We found proving this result to be a challenging problem. In what follows, we will assume that $n=9 m, m \geq 1$.

Let $\left(a_{i}, b_{i}, c_{i}\right)$ be an ordered set of integers. We call $\left(a_{i}, b_{i}, c_{i}\right)$ a triangular triple if it satisfies the following conditions:

a) $a_{i}, b_{i}$, and $c_{i}$ are all different,

b) $a_{i}+b_{i}+c_{i}=n-3$, and

c) $1 \leq a_{i}, b_{i}, c_{i} \leq \frac{n-3}{2}$.

Observe that for any vertex $p_{r}$ of $P$, a triangular triple $\left(a_{i}, b_{i}, c_{i}\right)$, defines a triangle $\Delta\left(p_{r}, p_{r+a_{i}+1}, p_{r+a_{i}+b_{i}+2}\right)$ of $P$. Moreover, condition c) above ensures that $\Delta\left(p_{r}, p_{r+a_{i}+1}, p_{r+a_{i}+b_{i}+2}\right)$ is acute, and hence it contains the center $c$ of $P$. Note that since $a_{i}+b_{i}+c_{i}=n-3, p_{r}=p_{r+a_{i}+b_{i}+c_{i}+3}$, addition taken $\bmod n$. Thus the edges of $\Delta\left(p_{r}, p_{r+a_{i}+1}, p_{r+a_{i}+b_{i}+2}\right)$ skip $a_{i}$, $b_{i}$, and $c_{i}$ vertices of $P$ respectively; see Figure 10 a).

Let $S\left(a_{i}, b_{i}, c_{i}\right)=\left\{\Delta\left(p_{r}, p_{r+a_{i}+1}, p_{r+a_{i}+b_{i}+2}\right): p_{r} \in P\right\}$. The set $S\left(a_{i}, b_{i}, c_{i}\right)$ can be seen as the set of triangles obtained by rotating $\Delta\left(p_{0}, p_{0+a_{i}+1}, p_{0+a_{i}+b_{i}+2}\right)$ around the center of $P$; see Figure $10 \mathrm{~b}$ ). The next observation will be useful:

Observation 2. Let $\left(a_{i}, b_{i}, c_{i}\right)$ and $\left(a_{j}, b_{j}, c_{j}\right)$ be triangular triples of $P$ such that $\left\{a_{i}, b_{i}, c_{i}\right\} \cap\left\{a_{j}, b_{j}, c_{j}\right\}=\emptyset, i \neq j$. Then all of the triangles in $S\left(a_{i}, b_{i}, c_{i}\right) \cup S\left(a_{j}, b_{j}, c_{j}\right)$ are edge-disjoint.

Consider a set $C=\left\{\left(a_{0}, b_{0}, c_{0}\right), \ldots,\left(a_{k-1}, b_{k-1}, c_{k-1}\right)\right\}$ of ordered triangular triples. We say that $C$ is a generating set of triangular triples if the following condition holds:

$$
\left\{a_{i}, b_{i}, c_{i}\right\} \cap\left\{a_{j}, b_{j}, c_{j}\right\}=\emptyset, i \neq j .
$$

Note that $\left|S\left(a_{i}, b_{i}, c_{i}\right)\right|=n$, and thus

$$
\bigcup_{\left(a_{i}, b_{i}, c_{i}\right) \in C} S\left(a_{i}, b_{i}, c_{i}\right)
$$

contains $n k$ edge disjoint triangles containing the center $P$. Our task is now that of finding a generating set of as many triangular triples as possible. 


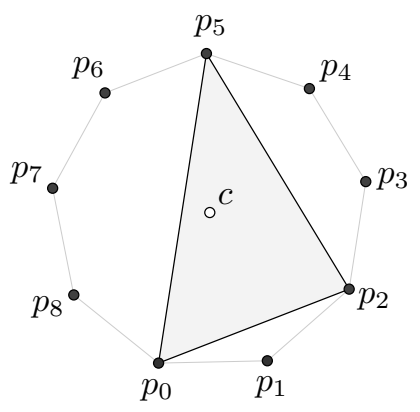

a)



b)

Figure 10: a) The triple $(1,2,3)$, and $p_{0}$ determine $\Delta\left(p_{0}, p_{2}, p_{5}\right)$. b) $S(1,2,3)$ is obtained by rotating $\Delta\left(p_{0}, p_{2}, p_{5}\right)$, obtaining a set of 9 edge-disjoint triangles.

389

390

391

Theorem 7. Let $P$ be the set of vertices of a regular polygon with $n=9 \mathrm{~m}$ vertices, and let $c$ be its center. Then if $m$ is odd, then $|\tau(c)| \geq \frac{n^{2}}{9}$, and if $m$ is even, then $|\tau(c)| \geq \frac{n^{2}}{9}-n$.

Proof. The proof when $m$ is odd proceeds by constructing a generating set $C$ with $\frac{n}{9}$ triangular triples. Let $k=\frac{9 m-3}{6}$ and $k^{\prime}=k+2 m-1$. Given $i \in\{0,1, \ldots, m-1\}$ we define the $i$-th ordered triple $\left(a_{i}, b_{i}, c_{i}\right)$ as follows (see Figure 11):

$$
\begin{aligned}
a_{i} & =k+i, \\
b_{i} & = \begin{cases}k^{\prime}-2 i-1 & \text { if } i<\frac{m-1}{2}, \\
k^{\prime}-2 i+m-1 & \text { if } i \geq \frac{m-1}{2}\end{cases} \\
c_{i} & = \begin{cases}k^{\prime}+i+1+\frac{m+1}{2} & \text { if } i<\frac{m-1}{2} \\
k^{\prime}+i+1-\frac{m-1}{2} & \text { if } \quad i \geq \frac{m-1}{2}\end{cases}
\end{aligned}
$$

We now prove that the triples $\left(a_{i}, b_{i}, c_{i}\right)$ are triangular; that is, $\mathrm{s} a_{i}+b_{i}+$ $c_{i}=n-3$. Since $b_{i}+c_{i}=2 k^{\prime}-i+\frac{m+1}{2}$ for all $i$,

$$
a_{i}+b_{i}+c_{i}=k+2 k^{\prime}+\frac{m+1}{2}=9 m-3 .
$$

It is easy to see that

$$
\begin{aligned}
k & \leq a_{i} \leq k+m-1, \\
k+m=k^{\prime}-m+1 & \leq b_{i} \leq k^{\prime}, \\
k^{\prime}+1 & \leq c_{i} .
\end{aligned}
$$






Figure 11: Triangular triples for $n=27,45,63,81$ and 99.



a)

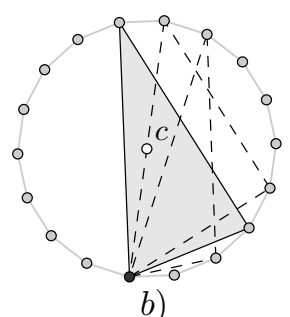

b)

Figure 12: a) Triangular triples $\left(a_{i}, b_{i}, c_{i}\right)$ for $n=9 \cdot 3=27$ and $\left.b\right)$ triples $\left(a_{i}^{\prime}, b_{i}^{\prime}, c_{i}^{\prime}\right)=\left(a_{i}-3, b_{i}-3, c_{i}-3\right)$ for $n=9 \cdot 2=18$.

392 Therefore $a_{i}<b_{j}<c_{k}$ for every $i, j, k$. Also, by construction it can be 393 verified that $a_{i} \neq a_{j}, b_{i} \neq b_{j}$, and $c_{i} \neq c_{j}$ for every $i \neq j$.

Thus the set $\bigcup_{\left(a_{i}, b_{i}, c_{i}\right) \in C}\left\{a_{i}, b_{i}, c_{i}\right\}$ contains no repeated elements.

394 Finally, note that the maximum value that can be reached by $c_{i}$ occurs 395 when $i=\frac{m-3}{2}$, and thus:

$$
c_{i} \leq k^{\prime}+1+\frac{m-3}{2}+\frac{m+1}{2}=k^{\prime}+m=\frac{9 m-3}{2} .
$$

396 Therefore $C$ is a generating set of triangular triples. Thus $c$ is contained 397 in at least $\frac{n^{2}}{9}$ edge-disjoint triangles. 
The proof when $m$ is even proceeds by also constructing a set of $m$ triples. We use the set of triples just constructed for $m+1$ and modify it as follows: Suppose that the set of $m+1$ triples is $\left\{\left(a_{0}, b_{0}, c_{0}\right), \ldots,\left(a_{m}, b_{m}, c_{m}\right)\right\}$.

Let $a_{i}^{\prime}=a_{i}-3, b_{i}^{\prime}=b_{i}-3$ and $c_{i}^{\prime}=c_{i}-3$ and consider $C^{\prime}=$ $\left\{\left(a_{i}^{\prime}, b_{i}^{\prime}, c_{i}^{\prime}\right) \mid 0 \leq i \leq m\right\}$. $C^{\prime}$ induces a set of triangles in $P$. Nevertheless $2 n$ triangles do not contain the point $c$ in their interior; see Figure 12. Therefore this construction guarantees that $c$ is contained in at least $(m+1) n-2 n=\frac{n^{2}}{9}-n$ edge-disjoint triangles.

\section{A point in many edge-disjoint empty triangles}

We conclude our paper by briefly studying the problem of the existence of a point contained in many edge-disjoint empty triangles of a point set. We point out that if we are interested only in empty triangles containing a point, it is easy to see that for any point set $P$, there is always a point $q$ contained in a linear number of (not necessarily edge-disjoint) empty triangles. This follows directly from the following facts:

1. Any point set $P$ with $n$ elements always determines at least a quadratic number of empty triangles $[2,16]$.

2. We can always choose $2 n-c-2$ points in the plane such that any empty triangle of $P$ contains one of them, where $c$ is the number of vertices of the convex hull of $P$; see $[8,16]$.

We now prove:

Theorem 8. There are point sets $P$ such that every $q \notin P$ is contained in at most a linear number of empty edge-disjoint triangles of $P$.

Proof. Let $H_{k}, H_{k-1}^{+}$and $H_{k-1}^{-}$be as defined in Section 2. Consider any set $T_{k}^{+}$(respectively $T_{k}^{-}$) of empty edge-disjoint triangles such that each of them has two vertices in $H_{k-1}^{+}$(respectively $H_{k-1}^{-}$) and the other in $H_{k-1}^{-}$ (respectively $H_{k-1}^{+}$). Let $t \in T_{k}^{+}$. Then the edge of $t$ with both endpoints in $H_{k-1}^{+}$is an edge of $H_{k-1}^{+}$visible from below. Since the triangles in $T_{k}^{+}$ are edge-disjoint, the number of elements of $T_{k}^{+}$is at most the number of edges of $H_{k-1}^{+}$visible from below, which is a linear function in $n$. Thus $\left|T_{k}^{+}\right| \in O(n)$. Similarly we can prove that $\left|T_{k}^{-}\right| \in O(n)$.

Consider a point $q \in \mathrm{CH}\left(H_{k}\right) \backslash \mathrm{CH}\left(H_{k-1}^{+}\right) \cup \mathrm{CH}\left(H_{k-1}^{-}\right)$. Clearly any empty triangle containing $q$ belongs to some $T_{k}^{+} \cup T_{k}^{-}$, and thus it belongs to at most a linear number of edge-disjoint triangles of $H_{k}$. 
Suppose next that $q \in \mathrm{CH}\left(H_{k-1}^{+}\right) \cup \mathrm{CH}\left(H_{k-1}^{-}\right)$. Suppose without loss of generality that $q \in \mathrm{CH}\left(H_{k-1}^{+}\right)$, and that $q$ belongs to a set $S$ of edgedisjoint triangles of $H_{k}$. S may contain some triangles with vertices in both of $H_{k-1}^{+}$and $H_{k-1}^{-}$. There are at most a linear number of such triangles. The remaining elements in $S$ have all of their vertices in $H_{k-1}^{+}$. Thus the number of edge-disjoint triangles containing $q$ satisfy

$$
T(n) \leq T\left(\frac{n}{2}\right)+\Theta(n),
$$

and thus $q$ belongs to at most a linear number of edge-disjoint triangles.

The first part of our result follows. To show that our bound is tight, let $q$ be as in the proof of Theorem 4. It is easy to see that $q$ belongs to a linear number of triangles with vertices in both of $H_{k}^{+}$and $H_{k}^{-}$, and our result follows.

We conclude with the following:

Conjecture 3. Let $P$ be a set of $n$ points in general position on the plane. Then there is always a point $q \notin P$ on the plane such that it is contained in at least $\log n$ edge-disjoint triangles of $P$.

\section{References}

[1] O. Aichholzer, R. Fabila-Monroy, D. Flores-Pealoza, T. Hackl, C. Huemer, and J. Urrutia. Empty monochromatic triangles. Computational Geometry, Theory and Applications, 42:934-938, 2009.

[2] I. Bárány and P. Valtr. Planar point sets with a small number of empty convex polygons. Studia Scientiarum Mathematicarum Hungarica, 41(2):243-266, 2004.

[3] C. Bautista-Santiago, M. Heredia, C. Huemer, A. Ramírez-Vigueras, C. Seara, and J. Urrutia. On the number of edges in geometric graphs without empty triangles. Submitted, January, 2011.

[4] E. Boros and Z. Füredi. The number of triangles covering the center of an $n$-set. Geom. Dedicata, 17:69-77, 1984.

[5] R. Buck and E. Buck. Equipartitions of convex sets. Math. Mag., 22:195-198, 1949. 
[6] B. Bukh. A point in many triangles. Electronic J. Combinatorics, 13(10), 2006.

[7] J. Ceder. Generalized sixpartite problems. Bol. Soc. Math. Mexicana, $2: 28-32,1964$.

[8] J. Czyzowicz, E. Kranakis, and J. Urrutia. Guarding the convex subsets of a point set. In Proc. 12th Canadian Conference on Computational Geometry, pages 47-50, 2000.

[9] K. Dehnhardt. Leere konvexe Vielecke in ebenen Punktmengen. Dissertation, TU Braunschweig, 1987.

[10] O. Devillers, F. Hurtado, G. Károlyi, and C. Seara. Chromatic variants of the Erdős-Szekeres Theorem. Computational Geometry Theory and Applications, 26(3):193-208, 2003.

[11] P. Erdős. Some more problems on elementary geometry. Austral. Math. Soc. Gaz., 5:52-54, 1978.

[12] P. Erdős and G. Szekeres. A combinatorial problem in geometry. Compositio Math., 2:463-470, 1935.

[13] T. Gerken. Empty convex hexagons in planar point sets. Discrete $\&$ Computational Geometry, 39(1-3):239-272, 2008.

[14] H. Harborth. Konvexe Fünfecke in ebenen Punktmengen. Elem. Math., $33: 116-118,1978$.

[15] J. D. Horton. Sets with no empty convex 7-gons. Canad. Math. Bull., 26:482-484, 1983.

[16] M. Katchalski and A. Meir. On empty triangles determined by points in the plane. Acta. Math. Hungar., 51:323-328, 1988.

[17] T. Kirkman. On a problem in combinatorics. Cambridge Dublin Math. J., 2:191-204, 1847.

[18] W. Morris and V. Soltan. The Erdős-Szekeres problem on points in convex position - a survey. Bulletin (new series) American Math. Soc., 37(4):437-458, 2000.

[19] C. M. Nicolás. The empty hexagon theorem. Discrete $\&$ Computational Geometry, 38:389-397, 2007. 
492

493

494

496

497

498

499

500

501

[20] J. Pach and G. Tóth. Monochromatic empty triangles in two-colored point sets. In Geometry, Games, Graphs and Education: the Joe Malkevitch Festschrift, pages 195-198, COMAP, Bedford, MA., 2008.

[21] G. Purdy. The minimum number of empty triangles. AMS Abstracts, 3:318, 1982.

[22] D. Ray-Chaudhuri and R. Wilson. Solution to Kirkman's schoolgirl problem. Proc. Sym. Pure Math. 19, Amer. Math. Soc., 19:187-204, 1971.

[23] H. J. Ryser. In Combinatorial Mathematics, pages 99-102, Buffalo, NY: Math. Assoc. Amer., 1963. 